Onkologie 1995;18:383

\title{
Impressum, Vol. 18, No. 5, 1995
}

Offizielles Organ der Deutsehen Geseüschaf’t für Hämatologie und Onkologie, der Osterreichischen Gesellschaft für Hämatologie und Onkologie, der Osterreichischen Krebsgesellschaft - Krebsliga unter Fortführung der «Österreichischen Zeitung für Onkologie» International Journal for Cancer Research and Treatment

Schriftleitung

W. Queißer, Mannheim H. Huber, Wien

Fachschriftleitung

W. E. Berdel, Berlin

E. Dühmke, München

R. Hartenstein, München

M. Kaufmann, Frankfurt/M.

H. Rübben, Essen

P. Schlag, Berlin

H.-J. Schmoll, Hannover

Wissenschaftlicher Beirat

L. Bergmann, Frankfurt/M.

C. Bokemeyer, Tubingen

V. Budach, Berlin

U. Creutzig, Münster

V. Diehl, Köln

P. Drings, Heidelberg

L. Edler, Heidelberg

G. Eisenbrand, Kaiserslautern

H. H. Fiebig, Freiburg

H. Gadner, Wien

C. Glanzmann, Zurich

R. Greiner, Bern

K. Havemann, Marburg F. Herrmann, Berlin R. Herrmann, Basel

D. Hoelzer, Frankfurt/M.

K. Höffken, Jena

H. J. Illiger, Oldenburg R. Jakesz, Wien U. R. Kleeberg, Hamburg R. Kreienberg, Ulm

M. Lahousen, Graz M. Lehnert, St. Gallen H. Löffler, Kiel H. Ludwig, Wien P. Lukas, Innsbruck H. J. Meyer, Hannover

F. A. Muthny, Münster

G. A. Nagel, Freiburg

A. Neiß, München

R. Parwaresch, Kiel

A. Pfleiderer, Freiburg

F Porzsolt, Ulm 


\author{
K. Possinger, Berlin \\ H. Sauer, München \\ G. Schackert, Dresden \\ R. Schulte-Hermann, Wien \\ S. Seeber, Essen \\ P. Sevelda, Wien \\ B. Thürlimann, St. Gallen \\ W. Tilgen, Heidelberg \\ M. Wannenmacher, Heidelberg H. J. Weh, Hamburg H. Wilke, Essen
}

Die Zeitschrift erscheint zweimonatlich; pro Jahr erscheint 1 Band zu je 6 Heften. Bezugspreisfür Jahrgang 18,1995, DM 173-/SFr 135,-, einschließlichMwSt, zu-züglich Postgebühren. Der Abonnementpreis ist im voraus zahlbar. Das Abonne-ment der Zeitschrift läuft weiter, wenn es nicht spätestens 4 Wochen vor Abschluß eines Bandes abbestell·t wird. Abonnementbestellungen können bei jeder Buchhandlung oder direkt beim Ver-lag aufgegeben werden:

Bundesrepublik Deutschland: S. Karger GmbH, Lörracher Str. 16 a, D-79115 Freiburg, Telefon (0761) 452070, Telefax (0761) 4520714, Postgiro München 40080-807

Übrige Lander: S. Karger AG, Allschwilerstr. 10, Postfach, CH-4009 Basel, Telefon (061)

3061111, Telefax (061) 3061234, E-Mail Karger@Karger.ch.

Anzeigen

S. Karger Verlag für Medizin und Naturwissenschaften GmbH,

Lörracher Str. 16 a, D-79115 Freiburg, Telefon (07 61) 452070.

Gültig ist die Preisliste Nr. 8 vom 1. Januar 1994.

Für den Inhalt außerhalb des redaktionellen Teiles (insbesondere Anzeigen, Industrieinformationen, Pressezitate und Kongreßinformationen) übernehmen Schriftleitung, Beirat und Verlag keine Gewähr.

Erne Markenbezeichnung kann warenzeichenrechtlich geschützt sein, auch wenn bei ihrer Verwendung in dieser Zeitschrift das Zeichen ${ }^{\circledR}$ oder ein anderer Hinweis auf etwa bestehende Schutzrechte fehlen sollte. Für Satzfehler, insbesondere bei Dosierungsangaben, wird keine Gewähr übernommen.

Die Zeitschrift sowie alle in ihr enthaltcnen einzelnen Beiträge und Abbildungen sind urheberrechtlich geschützt. Jede Verwertung, die nicht ausdrücklich vom Ur-heberrechtsgesetz zugelassen ist, bedarf der vorherigen Zustimmung des Verlags. Das gilt insbesondere für Vervielfältigungen, Bearbeitungen, Übersetzungen, Mi-kroverfilmungen und die Einspeicherung und Verarbeitung in elektronischen Sy-stemen. Fotokopien dürfen nur für den persönlichen Gebrauch als Einzelkopien hergestellt werden. Jede im Bereich eines gewerblichen Unternehmens zulässig hergestellte oder bcnutzte Kopie dient gewerblichen Zwecken gem. § 54(2) UrhG und verpflichtet zur Gebührenzahlung an die Verwertungsgesellschaft WORT, Abt. VG Wissenschaft, Goethestraße 49, D-80336 München 2.

(C) Copyright 1995 by S. Karger

Verlag für Medizin und Naturwissenschaften GmbH, Lörracher Str. 16 a

D-79115 Freiburg Verlagsleítung und presserechtlich verantwortlich: Sibylle Hopf Assistenz: Susanne Meister Herstellung: Georg Brunner Anzeigenverwaltung: Christiane Opitz 
Satz und Druck: Walter Biering GmbH Grafischer Betrieb Freisinger Landstraße 21 D-80939 München

KARGER 